\title{
Dislocation pinning effects on fracture behavior: Atomistic and dislocation dynamics simulations
}

\author{
S. J. Noronha and D. Farkas \\ Department of Materials Science and Engineering, Virginia Polytechnic Institute and State University, Blacksburg, Virginia 24061-0237
}

(Received 10 April 2002; published 8 October 2002)

\begin{abstract}
We introduce an approach in which results from atomistic simulations are combined with discrete dislocation dynamics simulations of crack-tip plasticity. The method is used to study the effects of dislocation pinning due to grain boundaries or secondary particles on the fracture behavior of aluminum. We find that the fracture resistance is reduced with decreasing pinning distance. The results show that the pinning of the dislocations causes a net decrease in the shear stress projected on the slip plane, preventing further dislocation emission. Semibrittle cleavage occurs after a certain number of dislocations is emitted.
\end{abstract}

DOI: 10.1103/PhysRevB.66.132103

PACS number(s): 62.20.Mk, 81.40.Np

Considerable attention has been devoted recently to the understanding of the crack-tip processes that are responsible for brittle versus ductile behavior in various materials including nanocrystalline metallic materials. There are two general prevailing types of models for assessing brittle-ductile crossover and transition behavior. The first type of conceptual model is based on the criterion for dislocation nucleation or emission at or in the vicinity of the crack tip: if a dislocation can be emitted from the crack tip prior to the cleavage propagation, it is considered that the material is ductile. The rationale is that the emitted dislocations blunt the crack, shield the crack tip from the applied external load, and therefore prevent crack propagation. The critical conditions for this to happen were obtained by several investigators starting with Kelly et al. ${ }^{1}$ A more recent significant development is due to Rice. ${ }^{2}$ Rice obtains the critical loadings for emission and cleavage by equating the crack-tip driving forces on distributed shear dislocation cores and cleavage opening, respectively, to the local resistance to dislocation propagation and cleavage along the slip/crack plane. The mode (either emission or further opening), which has equal driving force and resistance at the lowest applied load, determines the failure mode. The failure mode is controlled by two material parameters, the surface energy $\gamma_{s}$ and the so-called unstable stacking fault energy $\gamma_{u s}$.

According to these models, at a given temperature a material should be purely brittle or ductile. A similar conclusion is reached by Khantha et al. based on a model for the collective nucleation of dislocations. ${ }^{3}$ In reality most materials around the transition region show a mixed behavior of semiductile or semibrittle behavior. The second type of conceptual model accounts for this fact and is based on the concept of dislocation mobility: dislocations are emitted by sources activated by the high stresses at or near the crack tip and the rate at which they move away from the crack tip determines the material toughness. ${ }^{4,5}$ The emitted dislocations shield the crack tip and the source, inhibiting cleavage and further emission. However, the dislocations are mobile and hence move away from the crack tip under the influence of the local stress field, and as they move away more dislocations will be emitted. Experimental investigations have shown that dislocation mobility can be important in determining toughness. ${ }^{6}$ Atomistic simulations in $\mathrm{NiAl}$ have shown brittle behavior after the emission of sessile dislocations, also indi- cating the important role that dislocation mobility plays in toughness. ${ }^{7}$ This reasoning would indicate decreasing fracture toughness at very small grain sizes, because the grain boundaries hinder the motion of emitted dislocations. A different conclusion is reached if one reasons that a crack propagating within one grain no longer satisfies the fracture criterion when it crosses to the neighboring grain. The crack will thus be stopped more effectively, resulting in increased toughness, as the grain size decreases. For coarse grain systems, this latter prediction is observed in most cases, e.g., Ref. 8. The increase in fracture strength with decreasing grain size parallels a similar increase in yield strength with grain refinement, as given by the Hall-Petch relation, 9,10 which is usually explained on the basis of dislocation pileups at the grain boundary.

For nanocrystalline materials it has been shown that the Hall-Petch relation for yield strength fails at very low grain sizes and a breakdown of the Hall-Petch relation has been observed at grain sizes for which individual grains cannot support dislocation pile-up. ${ }^{11-13}$ At the smallest grain sizes, dislocation sources inside grains cannot exist as an intracrystalline deformation mechanism. Intercrystalline mechanisms such as grain boundary sliding become critical. Atomistic computer simulation studies of plastic deformation in nanocrystalline fcc materials have discussed this type of phenomenon. ${ }^{14-16}$ It is of great interest to explore the behavior of fracture toughness at very small grain sizes, and the effects of dislocations being absorbed or pinned by the large amount of grain boundaries can be very significant.

In this paper, we study the effects of dislocation pinning by obstacles near the crack tip such as grain boundaries in nanocrystalline materials. We combine atomistic and dislocation dynamics techniques to provide evidence that dislocation pinning can induce brittleness. We present results of dislocation pinning in $\mathrm{Al}$, using both discrete dislocation (DD) dynamics simulation and atomistic simulation. We find that pinning of emitted dislocations could substantially reduce the fracture toughness of ductile materials. The pinning can occur in the real material due to the presence of other defects, such as grain boundaries creating similar effects to those of low dislocation mobility.

In the simulations, we first performed an atomistic study of the crack-tip response and cleavage behavior as a function of the distance of the pinning point from the crack tip. Fail- 
ure is observed to occur at higher applied stress intensities, as the dislocation pinning distance is increased. Then the crack-tip plasticity is modeled around a semiinfinite crack using dislocation dynamics of two-dimensional (2D) discrete dislocation arrays. The fracture toughness and the number of dislocations emitted are found to increase with increasing pinning distance.

The details of the atomistic part of our simulation technique are as follows: We use a molecular statics technique with potentials based on an embedded atom method (EAM) developed for $\mathrm{Al}^{17}{ }^{17}$ The potentials were developed to reproduce the experimental properties of fcc $\mathrm{Al}$ as well as the properties of $\mathrm{Al}$ in several metastable crystal structures, as calculated using first principle linearized augmented plane wave (LAPW) methods. This helps to extend the validity of the EAM potential for configurations far from equilibrium. Blocks of about 10000 to about 200000 atoms are used. The boundary of the simulation size constitutes a fixed region that stops the propagation of the dislocations in the system. Each simulation size then represents a characteristic pinning distance for the dislocations, which is the distance from the crack tip to the boundary measured along the slip plane and perpendicular to the crack front. An initial atomically sharp crack is inserted with the crack tip at the middle of the simulation block. Periodic boundaries are imposed in the direction perpendicular to the crack front and the crack face. Displacement boundary conditions are used in the other two directions: the displacements of boundary atoms are fixed at the desired applied mode I stress intensity factor $K_{\text {Iapp }}$ according to the predictions of anisotropic elasticity theory. The interior atoms are then allowed to relax to a minimum energy configuration via a standard conjugate gradient relaxation technique. The applied stress intensity is increased slowly as to represent equilibrium quasistatic crack growth.

The crystallographic orientation of the crack is characterized by crack faces along a compact $\{111\}$ type plane and a crack front along a $\langle 110\rangle$ type direction. This geometry makes one slip plane of the $\{111\}$ type available for activation under mode I loading. Along this slip plane we observe emission of $\frac{1}{6}\langle 112\rangle$ Shockley partial dislocations. We observed dislocations emitted only in the forward direction, along the only slip plane available for emission under mode I loading. These dislocations begin to propagate away from the crack tip as the loading is increased, and eventually reach the boundaries of our simulation cell. A more detailed description of the atomistic simulation technique can be found in a previous paper. ${ }^{18}$

A simple 2D numerical dislocation dynamics (DD) model is employed for the present study. In the simulations, a semiinfinite crack without any dislocation is loaded from zero applied stress intensity. A dislocation source is assumed to exist at the crack tip, which emits dislocations along the slip plane. The elastic interaction of the dislocations emanating from the crack-tip region is described by isotropic elasticity, taking into account all self-interactions and image effects due to the free crack surfaces. ${ }^{19}$ The crack is loaded in mode I to an applied stress intensity $K_{\mathrm{I}}=K$. The in-plane and out-ofplane shear components are neglected $\left(K_{\mathrm{II}}=K_{\mathrm{III}}=0\right)$. The

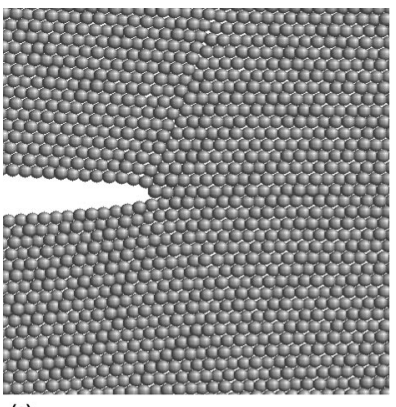

(a)

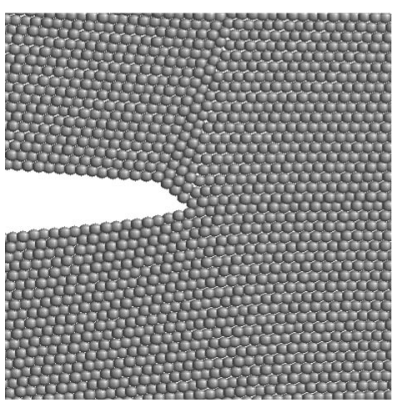

(b)

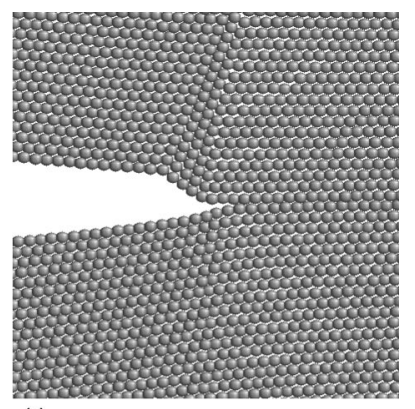

(c)

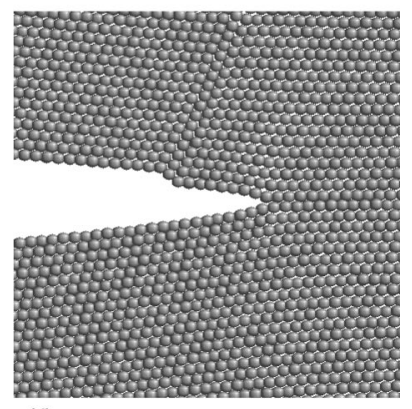

FIG. 1. Crack-tip configuration for different stresses (a), (b), (c), and (d) correspond to $0.95,1.6,1.8$, and 1.9 times the critical stress intensity for mode I fracture (Griffith value), respectively.

emitted dislocations are of edge character with their line direction parallel to the crack front, and Burgers vector parallel to the slip direction. The fracture is assumed to propagate when the crack-tip stress intensity reaches a critical value given by the Griffith criterion $(0.382 \mathrm{MPa} \sqrt{m})$. We took into account the change in crack-tip stress field due to the blunting of the crack tip when dislocations are emitted, and found the effect as insignificant when compared to the shielding contribution from the emitted dislocations. Since there is significant effect from crack blunting in the case of fcc metals, we have taken into account the crack blunting by changing the crack-tip stress field, with the emission of each dislocation. This was done by taking the crack-tip radius due to blunting as $n b \sin \theta$, where $n$ is the number of dislocations emitted, $b$ the Burgers vector, and $\theta$ the slip plane angle. This value of the crack-tip radius is then used in the expressions due to Creager and Paris ${ }^{20}$ to obtain the stress field. The dislocations are assumed to be emitted from the crack tip, and in the simulations this was represented by a source position at a distance of $1 b$ (the Burgers vector).

In order to have a correspondence between the DD modeling and the atomistic simulations we chose modeling parameters consistent with the crystallography of the atomistic simulation. In the atomistic simulations, when the crack is oriented in a $\{111\}$-type plane with a [110]-type crack front, the dislocations are emitted in adjacent inclined \{111\}-type planes. To mimic this we have chosen a slip plane $70.5^{\circ}$ tilted to the crack plane. The magnitude of Burger's vector is taken as $(b=a / \sqrt{6}, a=4.05 \AA)$.

In the dislocation dynamics simulations, the applied stress 

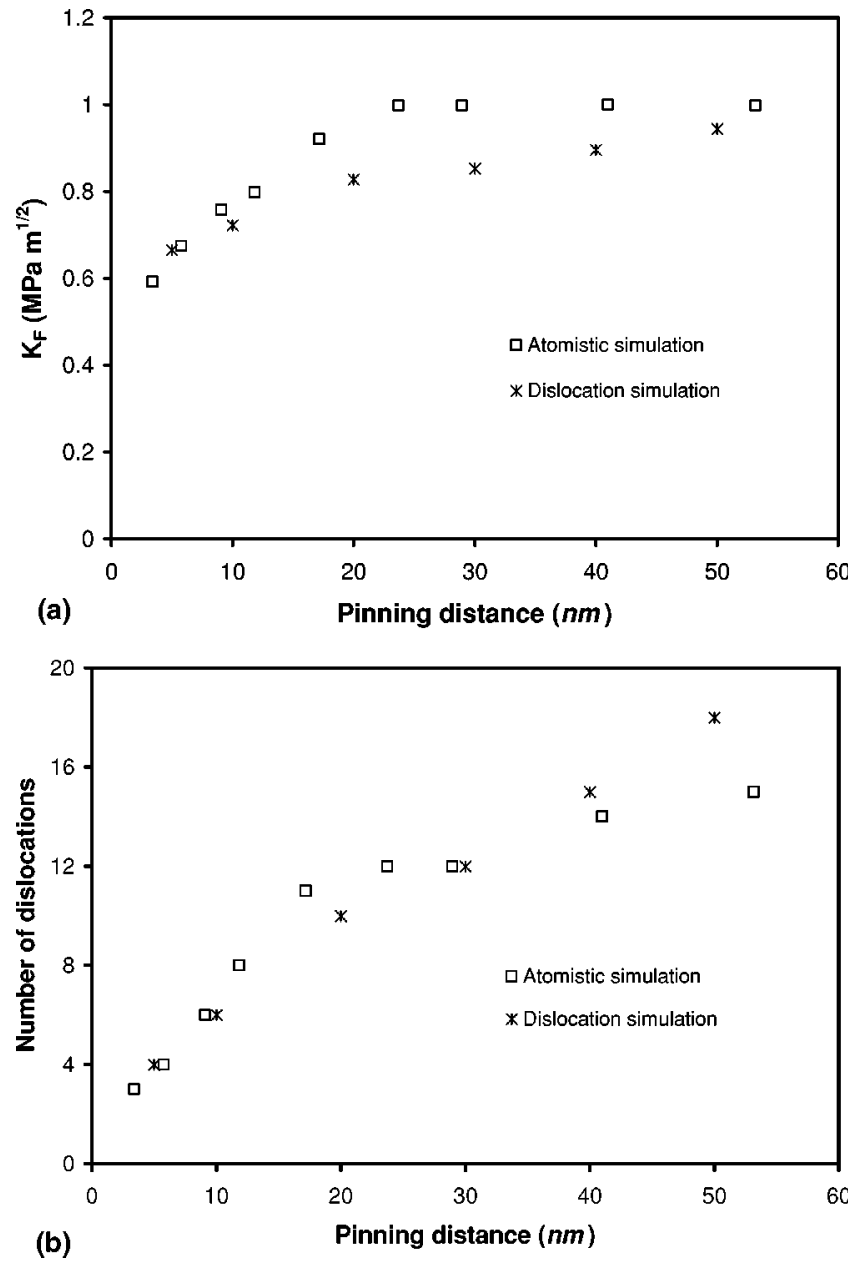

FIG. 2. Comparison of the atomistic and dislocation dynamics simulations, showing the total number of dislocations emitted as a function of pinning distance (b) and applied stress intensity at the reentry cleavage point (a).

intensity is increased at a constant rate, starting from zero, when there are no dislocations. At each time step a dislocation nucleation criterion is evaluated to decide whether a dislocation will be emitted. The emitted dislocations move along the slip plane based on an empirical velocity law derived from experimental results: ${ }^{21}$

$$
v=\frac{\tau b}{B(T)},
$$

where $v$ is the dislocation velocity which depends on the resolved shear stress along the slip plane $\tau . b$ is the magnitude of Burgers vector and $B(T)$ is the damping constant. ${ }^{21}$ At each time step, the total local stress intensity on the crack tip is calculated, as well as the total resolved shear stress on the slip plane. The nucleation of dislocations reduces the local stress intensity at the crack tip such that in the time step immediately before a new dislocation is nucleated, the local stress intensity is at its maximum. This maximum increases steadily in magnitude as the externally applied stress intensity is raised. Finally, the local total stress intensity $k$ at the
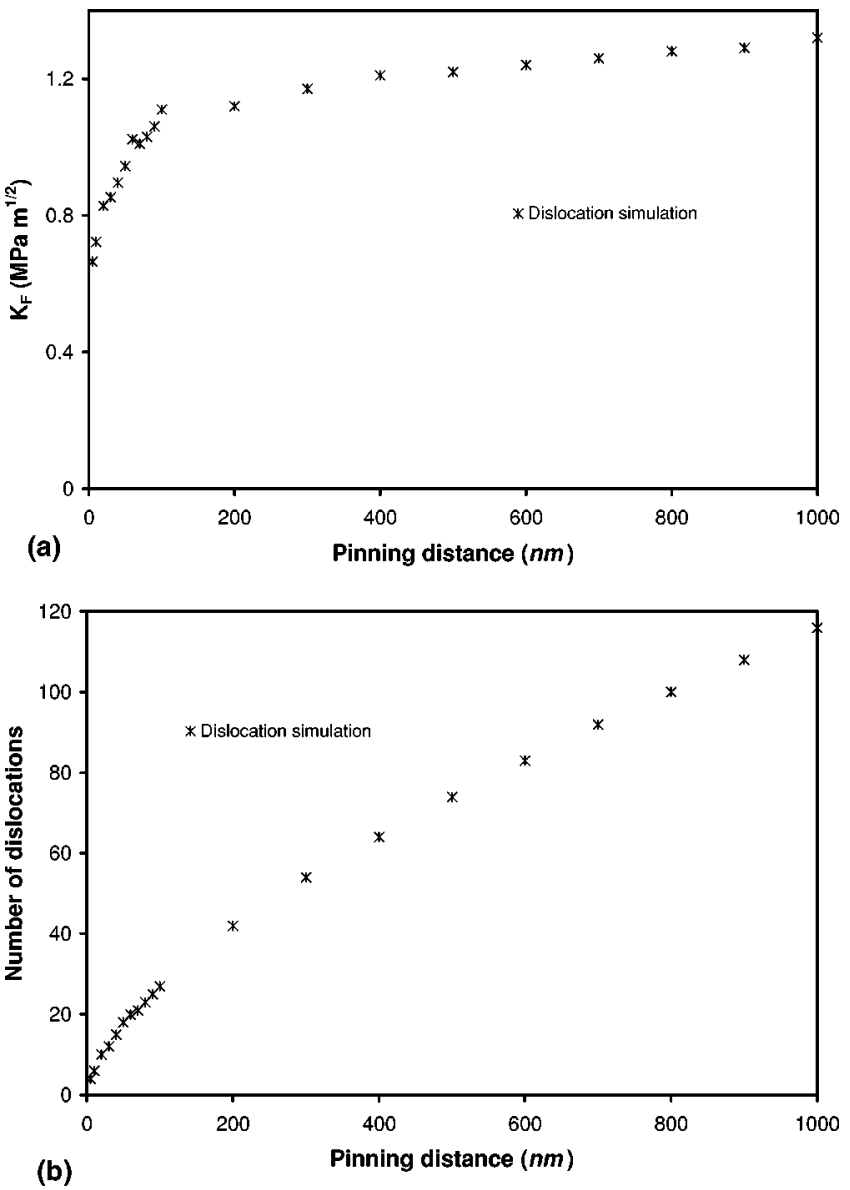

FIG. 3. (a) Fracture toughness obtained by dislocation emission as a function of pinning distance. (b) Number of dislocations emitted as function of pinning distance.

crack tip exceeds the critical value for fracture (here $K_{\mathrm{I} C}$ $=0.382 \mathrm{MPa} \sqrt{m})$. The applied stress intensity at this stage defines the point of reentry cleavage and therefore further crack propagation.

Figure 1 shows the atomic configuration of the crack-tip region as the external load is increased. These results show Shockley partial dislocations with Burgers vector $\frac{1}{6}\langle 112\rangle$ being emitted along inclined $\{111\}$-type planes, these dislocations are being emitted $70.5^{\circ}$ to the crack plane. At the first loading level (a) the first dislocation has just been emitted. The emission process continues in (b). In (c) reentry cleavage occurs and in (d) the crack has propagated further. These simulations are done using molecular statics and represent the configurations for which the system reaches equilibrium at each loading level.

Corresponding to the atomistic simulation, the dislocation simulation is done at a relatively low strain rate to allow for equilibrium to be reached. We used a strain rate of $0.04 \mathrm{MPa} \sqrt{\mathrm{ms}^{-1}}$ starting from $0.04 \mathrm{MPa} \sqrt{\mathrm{m}}$. This strain rate is slow enough for the dislocations to reach equilibrium configurations, and the results are not strain rate dependent in this regime. In Fig. 2 the external applied stress intensity and number of dislocations emitted at the reentry cleavage point is shown as a function of pinning distance. The figures show 
both the results of dislocation dynamics and atomistic simulations. For this range of pinning distances $(5 \mathrm{~nm}$ to $60 \mathrm{~nm}$ ) the values calculated using both length-scale methods show good agreement. There is also good agreement for the external applied stress intensity when the first dislocation is emitted, which is $0.28 \mathrm{MPa} \sqrt{\mathrm{m}}$ and $0.32 \mathrm{MPa} \sqrt{\mathrm{m}}$ in the dislocation dynamics and atomistic simulations, respectively. The critical stress intensity at emission resolved along the slip plane $\left(K_{\text {IIslip }}\right)$ calculated using the expression ${ }^{22}$

$$
K_{\text {IIslip }}=\frac{1}{2} \cos \left(\frac{\theta_{0}}{2}\right)\left[K_{\mathrm{I}} \sin \theta_{0}-K_{\mathrm{II}}\left(3 \cos \theta_{0}-1\right)\right]
$$

is found to be $0.1077 \mathrm{MPa} \sqrt{\mathrm{m}}$, for the case of DD simulation. This must be compared with the value of $0.1055 \mathrm{MPa} \sqrt{\mathrm{m}}$ obtained using $\gamma_{u s}$ values due to Rice $^{2}$ for Al.

Having been able to establish a correspondence between the atomistic and dislocation dynamics results, and their validity, we can now use dislocation dynamics to predict behavior for larger pinning distances, in a length scale that is not accessible to atomistics. These results are shown in Fig. 3. These results show that the trend of increasing ductility for increasing pinning distance continues up to larger pinning distances. If the pinning obstacles are grain sizes, this trend will continue to macroscopic grain sizes, for which one sees a ductile behavior. Experimental observation of significant loss (one order of magnitude) of ductility for nanocrystalline Al was reported. ${ }^{23}$ The fracture toughness values predicted by our model for coarse grained $\mathrm{Al}$ is less than the experimental values since our model does take in account dislocation multiplication by mechanisms such as Frank-Read sources, which will be activated for coarse grains. Due to this fact our model also fails to predict the Hall-Petch behavior shown by coarse grained materials. Full modeling of the fracture process in polycrystalline materials would also require a description of how grain boundaries can arrest a propagating crack. The effect of crack arrest by grain boundaries will be of an opposite nature to the effect studied in the present work. The modeling of the complete fracture process from first principles requires the accounting of interactions on widely different length scales. The motion of lattice dislocations, whose cores and Burgers vectors are on the atomic scale, generates plasticity. In pure solids, the dislocation velocity at low temperatures is controlled by dislocation-lattice interactions. However, plasticity requires multiplication of dislocations, which is controlled in general by dislocation sources, the stress to operate a source is controlled by elasticity. Here we attempted to learn the atomic processes from atomistic simulation and the parameters obtained are used in dislocation simulation to predict the behavior at large length scale.

We would like to thank W. A. Curtin, S. G. Roberts, and P. B. Hirsch for useful discussions. Part of the DD code was developed by S.J.N. during his stay at Oxford. The Office of Naval Research, Division of Materials Science, supported the work.
${ }^{1}$ A. Kelly, W.R. Tyson, and A.H. Cotrell, Philos. Mag. 15, 567 (1967).

${ }^{2}$ J.R. Rice, J. Mech. Phys. Solids 40, 239 (1992).

${ }^{3}$ M. Khantha, D.P. Pope, and V. Vitek, Phys. Rev. Lett. 73, 684 (1994).

${ }^{4}$ P.B. Hirsch and S.G. Roberts, Acta Mater. 44, 2361 (1996).

${ }^{5}$ P.B. Hirsch and S.G. Roberts, Philos. Trans. R. Soc. London, Ser. A 355, 1991 (1997).

${ }^{6}$ D. Caillard, C. Vailhe, and D. Farkas, Philos. Mag. A 79, 723 (1999).

${ }^{7}$ D. Farkas, Scr. Mater. 39, 553 (1998).

${ }^{8}$ D.A. Curry and J.F. Knott, Met. Sci. 12, 511 (1978).

${ }^{9}$ E.O. Hall, Proc. Phys. Soc. London, Sect. B 64, 747 (1951).

${ }^{10}$ N.J. Petch, J. Iron Steel Inst., London 174, 25 (1953).

${ }^{11}$ C.C. Koch, D.G. Morris, K. Lu, and A. Inoue, MRS Bull. 24, 54 (1999).

${ }^{12}$ J.R. Weertman, D. Farkas, K. Hemker, H. Kung, M. Mayo, R. Mitra, and H. Van Swygenhoven, MRS Bull. 24, 44 (1999).

${ }^{13}$ R.A. Masumura, P.M. Hazzledine, and C.S. Pande, Acta Mater.
46, 4527 (1998)

${ }^{14}$ H. Van Swygenhoven and A. Caro, Appl. Phys. Lett. 71, 1652 (1997).

${ }^{15}$ J. Schiotz, F.D. Di Tolla, and K.W. Jacobsen, Nature (London) 391, 561 (1998).

${ }^{16}$ V. Yamakov, D. Wolf, and M. Salazar, Acta Mater. 49, 2713 (2001).

${ }^{17}$ Y. Mishin, D. Farkas, M. Mehl, and D.A. Papaconstantopoulos, Phys. Rev. B 59, 3393 (1999).

${ }^{18}$ D. Farkas, M. Duranduru, W.A. Curtin, and C. Ribbens, Philos. Mag. A 81, 1241 (2001).

${ }^{19}$ V. Lakshmanan and J.C.M. Li, Mater. Sci. Eng., A 104, 95 (1988).

${ }^{20}$ M. Creager and P.C. Paris, Int. J. Fract. Mech. 3, 247 (1967).

${ }^{21}$ J.A. Gorman, D.S. Wood, and T. Vreeland, J. Appl. Phys. 40, 833 (1969).

${ }^{22}$ T.C. Wang, Philos. Mag. A 73, 981 (1996).

${ }^{23}$ E. Bonetti, L. Pasquini, and E. Sampaolesi, Nanostruct. Mater. 9, 611 (1997). 